\title{
Effects of a Modified Hand Compression Bandage for Treatment of Post-Burn Hand Edemas
}

\author{
Won Yong Park, $\mathrm{MD}^{1}$, Soo Jin Jung, $\mathrm{MD}^{1}$, So Young Joo, $\mathrm{MD}^{2}$, Ki Un Jang, $\mathrm{MD}^{2}$, \\ Cheong Hoon Seo, MD, $\mathrm{PhD}^{2}$, Ah Young Jun, $\mathrm{MD}$, $\mathrm{PhD}^{1}$
}

\begin{abstract}
${ }^{1}$ Department of Rehabilitation Medicine, Hallym University Dongtan Sacred Heart Hospital, Hwaseong;
${ }^{2}$ Department of Rehabilitation Medicine, Hallym University Hangang Sacred Heart Hospital, Seoul, Korea
\end{abstract}

Objective To evaluate the effect of a modified hand compression bandage in patients with a post-burn hand edema.

Methods Patients were recruited from burn centers. We classified the patients into two groups: the modified hand compression bandage group comprising of 22 patients who had a modified hand compression bandage and received conventional physical therapy and the conventionally treated group, comprising of 20 patients who received only conventional physical therapy during the 4 -week period post-burn. Hand circumference, hand skin thickness, and hand function were evaluated by grip strength, active range of motion (ROM), Jebsen hand function test, and visual analogue scale (VAS). These assessments were used to evaluate treatment effectiveness prior to the first treatment, 2 weeks after the first treatment, 4 weeks after the first treatment, and 4 months after the first treatment.

Results As a result of repeated-measures analysis of variance on hand circumference, skin thickness, VAS, and each metacarpophalangeal joint ROM, we found significant differences that corresponded to time effect $(\mathrm{p}<0.05)$ and timexgroup (reciprocal action) effect $(\mathrm{p}<0.05)$. The results of grasp power, Jebsen hand function test, and each proximal interphalangeal joint ROM, show significant differences in accordance with the time effect $(p<0.05)$, however, there was no reciprocal action effect $(\mathrm{p}>0.05)$.

Conclusion The modified hand compression bandage will be clinically useful for the treatment of patients with post-burn hand edemas.

Keywords Compression bandages, Burns, Edema, Rehabilitation

Received September 3, 2015; Accepted September 16, 2015

Corresponding author: Soo Jin Jung

Department of Rehabilitation Medicine, Hallym University Dongtan Sacred Heart Hospital, 7 Keunjaebong-gil, Hwaseong 18450, Korea Tel: +82-31-8086-2350, Fax: +82-31-8086-2317, E-mail: werch@hallym.or.kr

(c) This is an open-access article distributed under the terms of the Creative Commons Attribution Non-Commercial License (http://creativecommons.org/ licenses/by-nc/4.0) which permits unrestricted noncommercial use, distribution, and reproduction in any medium, provided the original work is properly cited. Copyright $\odot 2016$ by Korean Academy of Rehabilitation Medicine 


\section{INTRODUCTION}

The most common body parts where burns occur are the hands and arms. The main complications of hand burns are edemas, which are the pathological accumulation of the fluid in the extracellular space [1]. Hand edema is followed by the aggravation of symptoms, such as swelling and pain, and major complications including finger joint contracture. Edema onset begins immediately after burn injury and continues to enlarge up to 2-3 weeks later. Therefore, muscle weakness and disturbance of joint structure can result from edema and when coupled with immobilization of movement, it contribute to the formation of adhesion and disturbances in the normal sliding and gliding motion between the underlying structures. In absence of therapeutic exercise, secondary changes in the articular and supporting structures will result in joint stiffness and decrease joint excursion with deterioration of function [2].

For hand burn patients, a resting hand splint with the inclusion of wrist extension or compression garment is used to prevent edema, contracture and deformity $[3,4]$. It is generally known that resting hand splint with the inclusion of wrist extension is applied to patients with acute post-burn hand edemas at the burn center in the early stage of injury. Moreover, a compression garment is applied in such a condition that there is scar maturation after complete wound healing in the stage of physical therapy. The problems of compression garment are great discomfort, blistering, wound breakdown, pruritus, and uncontrolled pressure [5]. Moreover, there is a low compliance problem due to the difficulty in wearing a compression garment. Further, the range of motion (ROM) exercise is impaired due to the pressure applied to the joint area when wearing the compression garment. Nevertheless, the most adverse problem concerning the postburn bandaging is that the compression garment can only be applied after wound healing and scar maturation and cannot be applied easily in the early stage of burn injury [5]. For this reason our burn center used a compression bandage, similar to those bandages used for breast cancer treatment-related lymphedema, to patients with a post-burn hand edema.

To ensure that patients can perform the ROM exercise, we used a novel type of modified hand compression bandage with in the first stage bandaging, avoiding the proximal interphalangeal (PIP) joint and metacarpophalangeal (MCP) joint. Thus, we treated patients with early onset burn injury that occurred within 3 months and for whom a compression garment could not be applied.

The primary objective of this study was to evaluate the circumference effectiveness in applying a modified hand compression bandage to patients with post-burn hand edemas. The secondary objective of this study was to evaluate the modified bandage's effectiveness in visual analogue scale (VAS), ROM, grasps power, hand function, and skin thickness.

\section{MATERIALS AND METHODS}

This is a retrospective cohort study, reviewed at Institutional Review Board. All consecutive patients who were admitted to the special burn center from January 2012 to December 2014 and treated for post-burn hand injury were identified by a retrospective chart review. Included were burn patients who had symptoms of aggressively and rapidly progressing hand swelling, whose hands had a deep partial-thickness (second-degree deep) burn or full-thickness (third-degree) burn, contracture of joint, pain transferred to the rehabilitation department after acute burn treatment, and less than 3 months since the onset of burn injury. Exclusion criteria were severe wound infection or cellulitis, musculoskeletal diseases (fractures, amputation, rheumatoid arthritis, and degenerative joint disease), nervous diseases (peripheral nerve disorder, cervical radiculopathy), and electrical burn patients.

The patients were classified into two groups: the modified hand compression bandage group who had a modified hand compression bandage applied and had received conventional physical therapy and the conventional treated group who received only conventional physical therapy. The modified hand compression bandaging was applied to the patients who were treated by a physical therapist 6 days a week for 4 weeks. A modified hand compression bandage was applied to make it possible to perform the ROM exercise at the MCP and PIP joint, while avoiding the bandaging in the joint areas.

The method to apply the modified hand compression bandage firstly uses a one inch bandage gauze (miro Verbandstoffe $\mathrm{GmbH}$, Wiehl, Germany) to wrap from the wrist to all the 5 fingers, initiating the fingertip to 

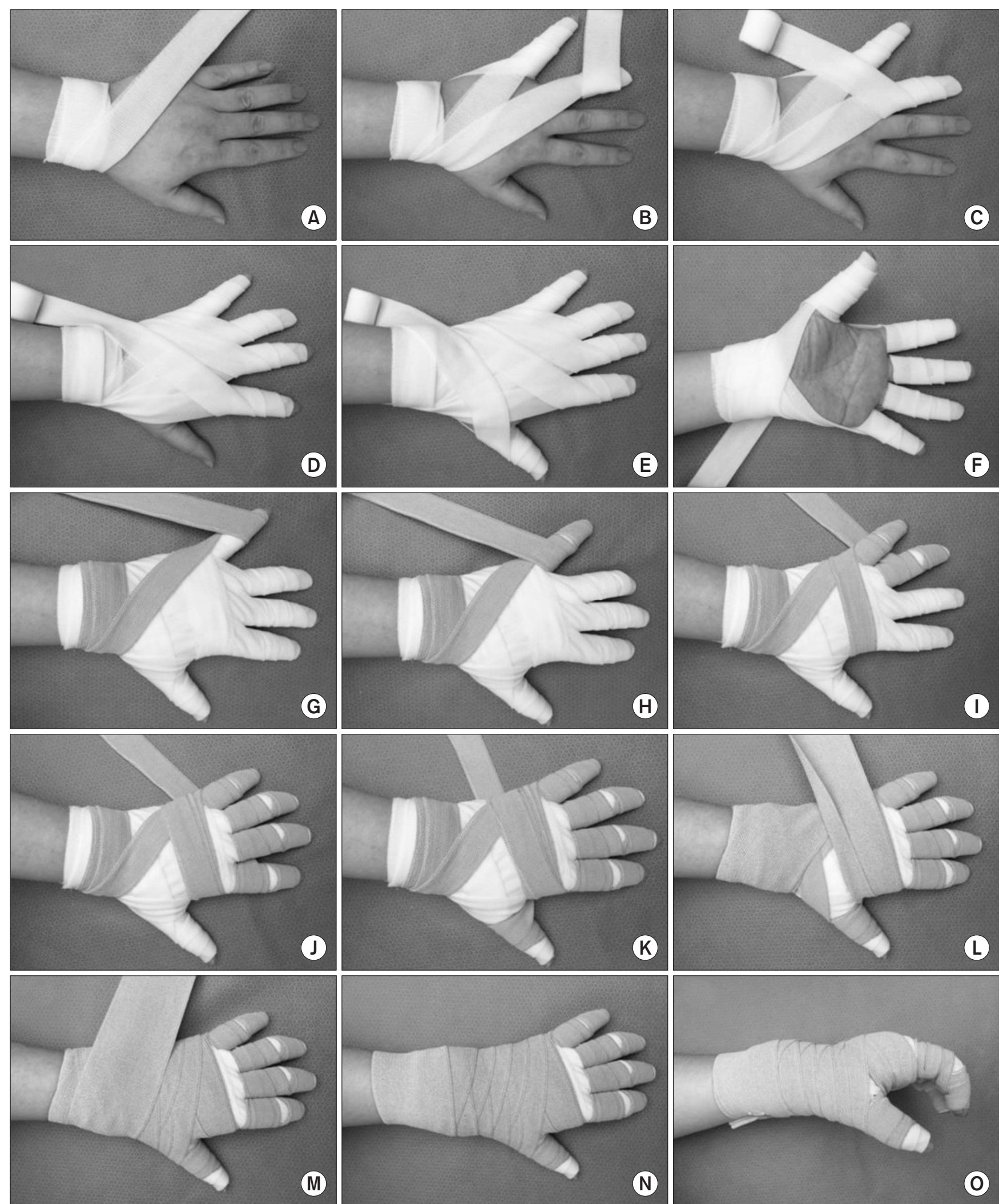

Fig. 1. (A-O) Method of applying modified hand compression bandage. 

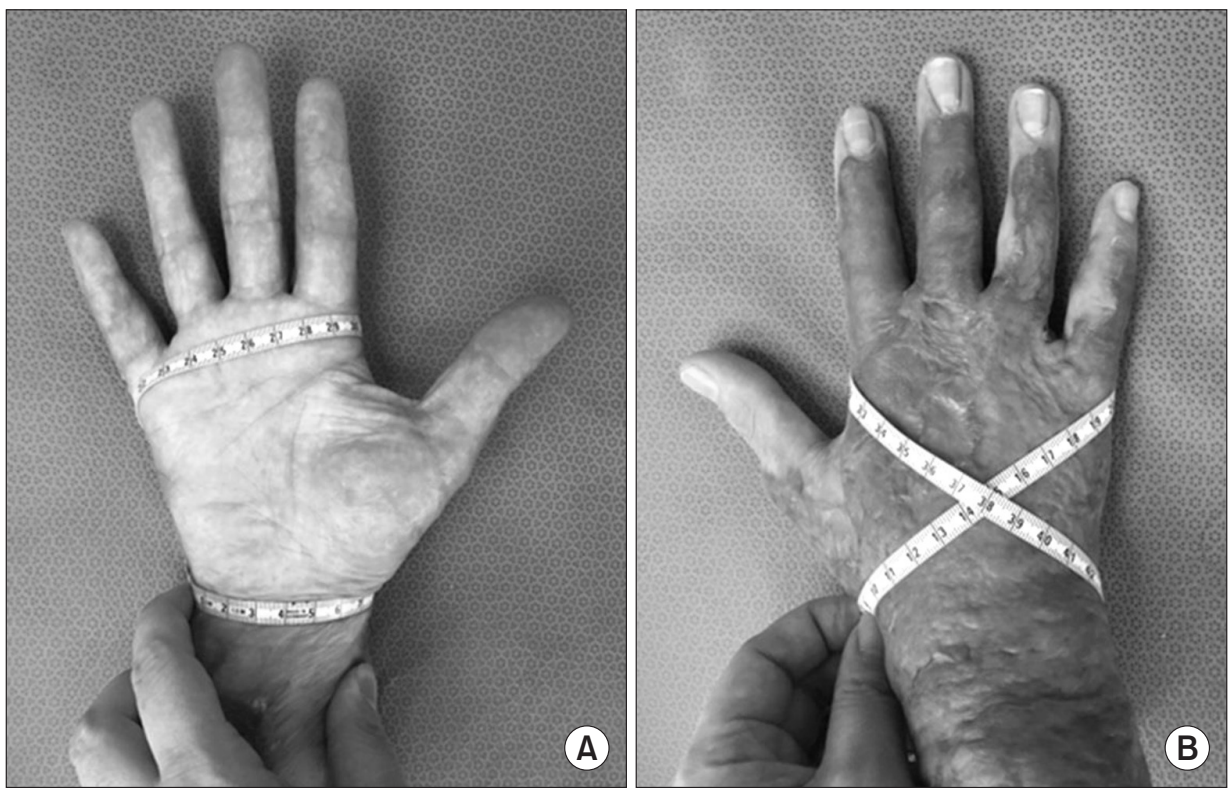

Fig. 2. Placement of tape measure for figure-eight method of measuring hand circumference, ventral surface (A) and dorsal surface of the hand (B). First, the zero mark of tape measure was placed over the distal aspect of the ulnar styloid process, and the tape was drawn across the ventral surface of the wrist to the distal aspect of the radial styloid process. Next, the tape measure was drawn diagonally across the dorsum of the hand, brought over the ventral surface of the metacarpophalangeal joints, and wrapped diagonally across the dorsum to return to the starting point.

the MCP joint. Thereafter, a one inch short stretch bandage (miro Verbandstoffe) was used to wrap all 5 fingers, excluding the MCP and PIP joint. Last, bandaging was performed from the MCP joint to the wrist, using a three inch short stretch bandage (miro Verbandstoffe) (Fig. 1).

All patients had four weeks of intensive rehabilitation and hospitalization. After their discharge, the both groups had standard rehabilitation and wore compression garment to the outpatient clinic. Both groups received the standard rehabilitation therapy focusing on post-burn hand therapy such as passive ROM exercise, activities of daily living training, manual lymphatic drainage, and skin care.

The following data were collected from the case records: the hand size using the figure-eight method, grip strength, the MCP joint ROM, the PIP joint ROM, skin thickness using ultrasonography, Jebsen hand function test, and VAS $[4,6]$.

For the figure-eight tape method protocol, the following procedures were performed. 1) The starting point for the tape measure was the outermost aspect of the most distal aspect of the ulnar styloid process. 2) The tape measure was drawn across the ventral surface of the wrist to the most distal aspect of the radial styloid process. 3) The tape measure was placed diagonally across the dorsal aspect of the hand with the proximal surface of the tape measure placed over the fifth MCP joint line. 4) The tape measure was then brought across the ventral surface of the MCP joints and the proximal surface of the tape mea-

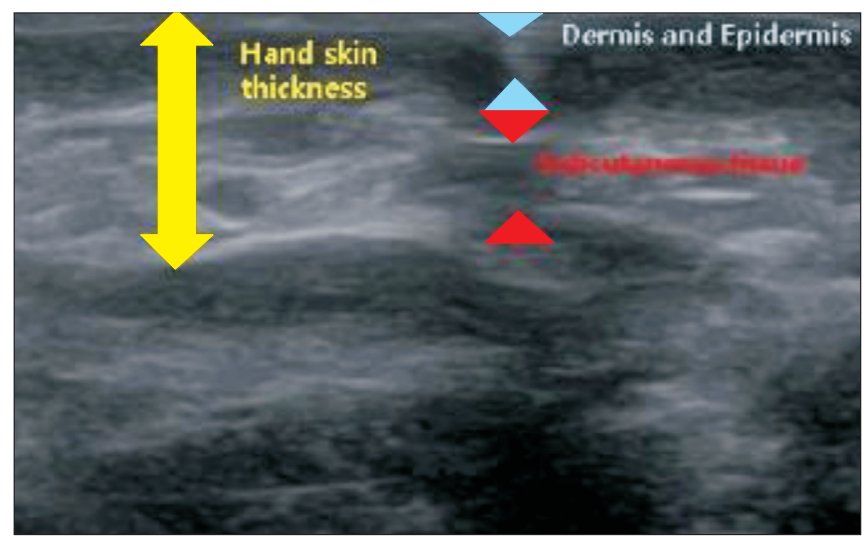

Fig. 3. Hand thickness measured by using ultrasonography. ${ }^{*} \mathrm{p}<0.05$, independent $\mathrm{t}$-test.

sure positioned over the second MCP joint line. 5) The tape measure was wrapped diagonally across the dorsum of the hand back to the starting point [7,8] (Fig. 2).

The active joint motion range and the grip strength were measured using the goniometer (Jamar StainlessSteel Finger Goniometers; Lafayette Instrument, Lafayette, IN, USA) and squeeze dynamometer (Lafayette Instrument), respectively. These tests evaluated the changes in the flexion limit of MCP joint and PIP joint.

The hand skin thickness measured the sum of skin and subcutaneous tissue thicknesses using ultrasonography (Medison, Seoul, Korea) (Fig. 3). Since each patient has burn wound in different location, the site with thickest skin in wound area was chosen and marked for measure 
[9]. Four tests were conducted after recording the initial measurement and taking photos of the exact location each time. The Jebsen hand function test was performed by one occupational therapist. In addition, a designated physician measured VAS was in a resting state by [10].

Each scale was assessed prior to the first treatment (baseline assessment), 2 weeks after the first treatment, 4 weeks after the first treatment, and 4 months after the first treatment. Data collected were analyzed by using the SPSS ver. 18.0 program (SPSS Inc., Chicago, IL, USA). An independent t-test was carried out for homogeneity tests in both groups. Repeated-measures analysis of variance (ANOVA) was carried out to examine the effectiveness of the results. A significant difference in accordance with timexgroup (reciprocal) effect showed the effectiveness of the treatment. Additional analyses were performed to compare the differences depending on the time point between the two groups, for which we used and independent t-test.

\section{RESULTS}

The modified hand compression bandage group and the conventionally treated groups were comprised of 22 patients (18 men and 4 women) and 20 patients (14 men and 6 women), respectively. The mean age was $45.86 \pm 8.51$ years old in the modified hand compression bandage group and $45.85 \pm 5.73$ years old in the conventionally treated group.

Using the independent t-test, both groups were found to be homogeneous as there was no significant difference ( $p>0.05$ ). Moreover, there was no significant difference in baseline assessment data between the two groups ( $p>0.05)$ (Table 1).

On repeated-measures ANOVA, variables considered for significant time effects $(\mathrm{p}<0.05)$ and reciprocal effects $(\mathrm{p}<0.05)$ were figure-eight hand circumference, skin thickness, VAS, and each MCP joint ROM. However, variables with significant time effects $(\mathrm{p}<0.05)$, but insignificant reciprocal effects $(\mathrm{p}>0.05)$ were grasp power,

Table 1. Demographic and clinical characteristics of participants

\begin{tabular}{lcc}
\hline & Modified hand compression bandage group & Conventional treated group \\
\hline Sex (male:female) & $18: 4$ & $14: 6$ \\
\hline Burn side (left:right) & $9: 13$ & $10: 10$ \\
\hline Age (yr) & $45.86 \pm 8.51$ & $45.85 \pm 5.73$ \\
TBSA (\%) & $31.91 \pm 19.88$ & $36.60 \pm 14.36$ \\
\hline Burn type (CoB:FB:SB:SPB) & $0: 19: 0: 3$ & $1: 17: 2: 0$ \\
\hline
\end{tabular}

Values are presented as mean \pm standard deviation.

TBSA, total burn surface area; CoB, contact burn; FB, flame burn; SB, scalding burn; SPB, spark burn.

Table 2. Metacarpophalangeal (MCP) joint range of motion

\begin{tabular}{lllccccc}
\hline MCP joint & Group & Baseline & \multicolumn{1}{c}{ 2 wk } & \multicolumn{1}{c}{ 4 wk } & 4 mo & \multicolumn{2}{c}{ p-value } \\
\hline Thumb & Bandage & $26.45 \pm 8.81$ & $38.14 \pm 6.77$ & $49.36 \pm 6.60$ & $59.41 \pm 4.08$ & $\mathrm{~T}$ & $<0.001$ \\
& Conventional & $28.40 \pm 9.16$ & $32.65 \pm 9.23$ & $35.85 \pm 10.10$ & $38.95 \pm 8.74$ & $\mathrm{G} \times \mathrm{T}$ & $<0.001$ \\
\multirow{2}{*}{ 2nd } & Bandage & $37.41 \pm 15.19$ & $50.36 \pm 12.74$ & $66.45 \pm 11.11$ & $80.77 \pm 7.91$ & $\mathrm{~T}$ & $<0.001$ \\
& Conventional & $30.55 \pm 16.38$ & $36.50 \pm 13.42$ & $44.75 \pm 14.00$ & $49.00 \pm 13.32$ & $\mathrm{G} \times \mathrm{T}$ & $<0.001$ \\
\hline \multirow{2}{*}{ 3rd } & Bandage & $40.77 \pm 15.37$ & $56.18 \pm 12.78$ & $72.91 \pm 10.97$ & $82.64 \pm 8.78$ & $\mathrm{~T}$ & $<0.001$ \\
& Conventional & $41.60 \pm 20.16$ & $53.35 \pm 17.70$ & $61.55 \pm 16.94$ & $66.45 \pm 16.45$ & $\mathrm{G} \times \mathrm{T}$ & $<0.001$ \\
\hline \multirow{2}{*}{ 4th } & Bandage & $41.41 \pm 15.31$ & $56.91 \pm 12.99$ & $72.27 \pm 9.31$ & $82.86 \pm 6.31$ & $\mathrm{~T}$ & $<0.001$ \\
\hline \multirow{2}{*}{5 th } & Conventional & $41.25 \pm 17.62$ & $46.20 \pm 16.22$ & $50.40 \pm 17.62$ & $51.50 \pm 17.68$ & $\mathrm{G} \times \mathrm{T}$ & $<0.001$ \\
& Bandage & $35.77 \pm 17.89$ & $51.14 \pm 13.09$ & $66.82 \pm 13.15$ & $81.14 \pm 10.35$ & $\mathrm{~T}$ & $<0.001$ \\
\hline
\end{tabular}

Values are presented as mean \pm standard deviation.

$\mathrm{G}$, group; $\mathrm{T}$, time. 
Table 3. Proximal interphalangeal (PIP) joint range of motion

\begin{tabular}{llccccrr}
\hline PIP joint & Group & Baseline & 2 wk & 4 wk & 4 mo & \multicolumn{2}{c}{ p-value } \\
\hline Thumb & Bandage & $30.68 \pm 20.82$ & $37.18 \pm 19.20$ & $42.14 \pm 20.63$ & $45.55 \pm 20.47$ & $\mathrm{~T}$ & $<0.001$ \\
& Conventional & $30.75 \pm 14.20$ & $34.80 \pm 14.51$ & $39.80 \pm 15.82$ & $44.25 \pm 15.62$ & $\mathrm{G} \times \mathrm{T}$ & 0.422 \\
\multirow{2}{*}{ 2nd } & Bandage & $53.00 \pm 19.45$ & $58.14 \pm 19.22$ & $69.55 \pm 22.38$ & $72.45 \pm 22.32$ & $\mathrm{~T}$ & $<0.001$ \\
\multirow{2}{*}{ 3rd } & Conventional & $51.70 \pm 28.72$ & $62.05 \pm 23.64$ & $71.00 \pm 23.22$ & $76.60 \pm 22.15$ & $\mathrm{G} \times \mathrm{T}$ & 0.348 \\
\hline \multirow{2}{*}{ 4th } & Bandage & $49.23 \pm 17.95$ & $56.59 \pm 18.77$ & $63.00 \pm 20.78$ & $67.18 \pm 18.95$ & $\mathrm{~T}$ & $<0.001$ \\
& Conventional & $47.25 \pm 23.41$ & $52.70 \pm 22.38$ & $60.40 \pm 23.12$ & $66.45 \pm 23.11$ & $\mathrm{G} \times \mathrm{T}$ & 0.561 \\
\hline \multirow{2}{*}{ 5th } & Bandage & $54.00 \pm 15.87$ & $60.32 \pm 14.99$ & $68.32 \pm 13.34$ & $70.55 \pm 14.05$ & $\mathrm{~T}$ & $<0.001$ \\
& Conventional & $46.05 \pm 23.32$ & $49.90 \pm 24.58$ & $52.80 \pm 26.78$ & $53.65 \pm 27.61$ & $\mathrm{G} \times \mathrm{T}$ & 0.251 \\
\hline & Bandage & $45.00 \pm 22.89$ & $58.14 \pm 16.93$ & $68.64 \pm 16.80$ & $76.32 \pm 16.21$ & $\mathrm{~T}$ & $<0.001$ \\
\hline
\end{tabular}

Values are presented as mean \pm standard deviation.

G, group; T, time.

Table 4. The change of figure-eight hand circumference, skin thickness, VAS, grasp power, Jebsen hand function test total scores

\begin{tabular}{llrrrrrr}
\hline \multicolumn{1}{c}{ Parameter } & \multicolumn{1}{c}{ Group } & Baseline & 2 wk & 4 wk & 4 mo & & p-value \\
\hline Circumference $(\mathrm{cm})$ & Bandage & $46.83 \pm 3.23$ & $44.20 \pm 3.56$ & $42.77 \pm 4.15$ & $40.21 \pm 2.32$ & $\mathrm{~T}$ & $<0.001$ \\
& Conventional & $47.20 \pm 4.23$ & $46.05 \pm 4.02$ & $45.50 \pm 4.13$ & $40.45 \pm 2.39$ & $\mathrm{G} \times \mathrm{T}$ & $<0.001$ \\
Skin thickness $(\mathrm{cm})$ & Bandage & $0.4970 \pm 0.16$ & $0.3877 \pm 0.12$ & $0.2955 \pm 0.10$ & $0.2700 \pm 0.09$ & $\mathrm{~T}$ & $<0.001$ \\
& Conventional & $0.4975 \pm 0.23$ & $0.4385 \pm 0.16$ & $0.3950 \pm 0.13$ & $0.2560 \pm 0.07$ & $\mathrm{G} \times \mathrm{T}$ & $<0.001$ \\
\multirow{2}{*}{ VAS } & Bandage & $7.07 \pm 1.53$ & $5.40 \pm 1.35$ & $4.13 \pm 1.06$ & $2.87 \pm 0.83$ & $\mathrm{~T}$ & $<0.001$ \\
& Conventional & $7.27 \pm 1.49$ & $6.40 \pm 1.18$ & $5.40 \pm 0.91$ & $5.80 \pm 2.01$ & $\mathrm{G} \times \mathrm{T}$ & $<0.001$ \\
Grasp power (kg) & Bandage & $5.52 \pm 4.74$ & $9.89 \pm 5.77$ & $13.14 \pm 7.33$ & $19.32 \pm 8.04$ & $\mathrm{~T}$ & $<0.001$ \\
& Conventional & $6.23 \pm 5.72$ & $9.50 \pm 6.51$ & $12.30 \pm 7.63$ & $17.35 \pm 7.95$ & $\mathrm{G} \times \mathrm{T}$ & 0.178 \\
\multirow{2}{*}{ Hand function test } & Bandage & $157.01 \pm 62.20$ & $122.84 \pm 45.84$ & $96.14 \pm 34.59$ & $73.32 \pm 21.35$ & $\mathrm{~T}$ & $<0.001$ \\
\hline & Conventional & $142.64 \pm 93.84$ & $12.75 \pm 74.31$ & $110.55 \pm 67.86$ & $74.17 \pm 32.90$ & $\mathrm{G} \times \mathrm{T}$ & 0.107 \\
\hline
\end{tabular}

Values are presented as mean \pm standard deviation.

VAS, visual analogue scale; $G$, group; $T$, time.

the Jebsen hand function test, and each PIP joint ROM (Tables 2-4, Fig. 4).

The ndependent t-test was performed to compare the time point difference between the two groups, figureeight hand circumference, and VAS showed a significant difference for 2 weeks and 4 weeks after treatment. Four months after treatment, there were no significant differences between the two groups. The skin thickness test showed a significant difference in the 4 weeks after treatment time point. The second and fourth MCP joint ROM showed a significant difference from 2 weeks after treatment to end of the study. The first and third MCP joint ROM showed a significant difference from 4 weeks after treatment to end of the study. The fifth MCP joint
ROM showed a significant difference up to 4 months after treatment. Grasp power, the Jebsen hand function test, and PIP joint ROM showed no significant difference during the study (Tables 2-4, Fig. 4).

\section{DISCUSSION}

Various complications occur after hand burn injury, which can result in limitations in everyday movements and in functional disorders $[11,12]$. Edema occurs even in the early stage of burn injury, alters the fibrosis of muscle, tendon and skin, and impairs the joint structures, thus, inducing the occurrence of complications that are exacerbated in time. Thus, early edema formation reduc- 
(A)

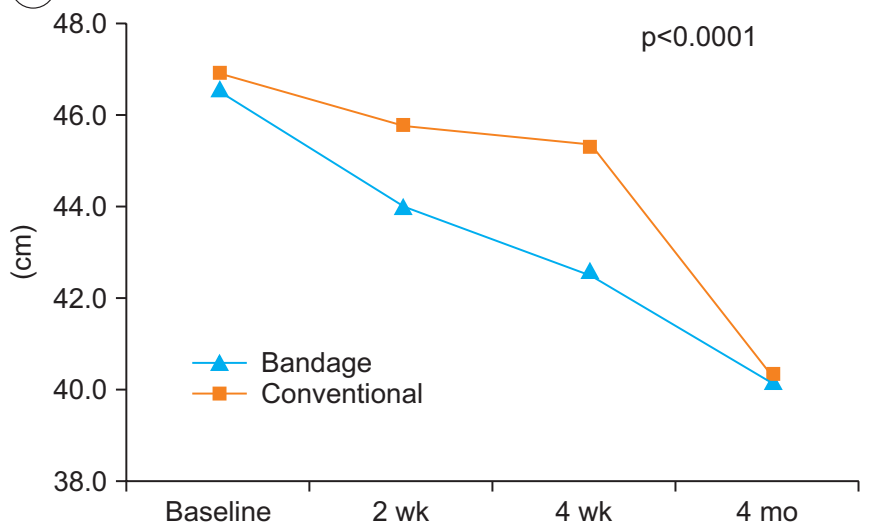

(C)

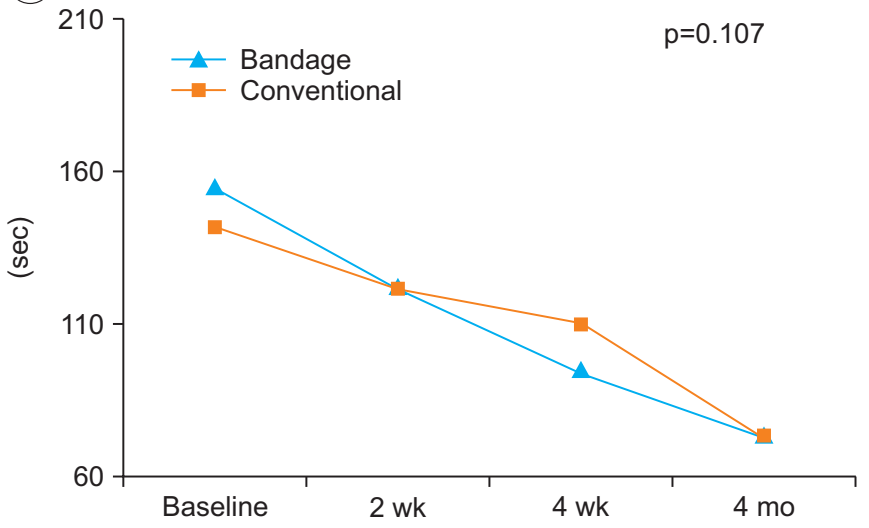

(E)

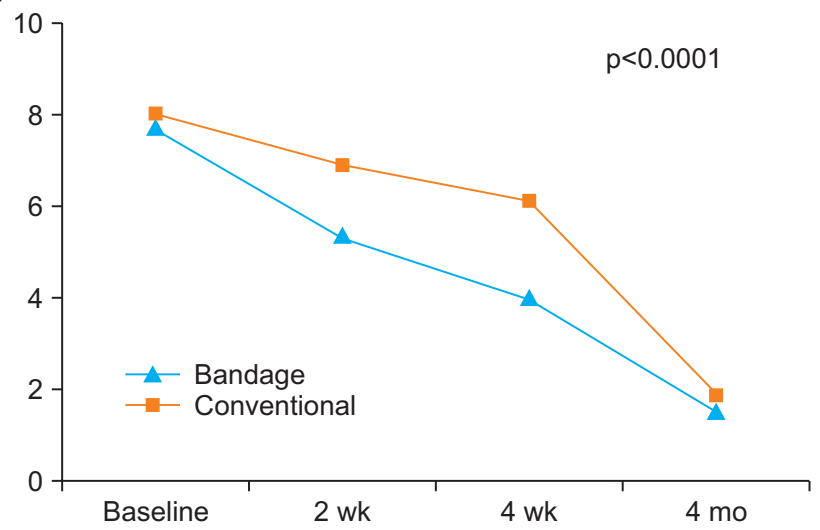

tion would prevent these complications.

Most of burn types such as contact burn, flame burn, scalding burn, spark burn, and electrical burn entail edema formation. The magnitude of post-burn edema is dependent on the depth of burn injury and heat exposure time, not burn type, with the exception of electrical burn [13].

Edema control through externally applied pressure is frequently used in burn centers and is considered clinically useful, although little objective data exist to sub-
(B)

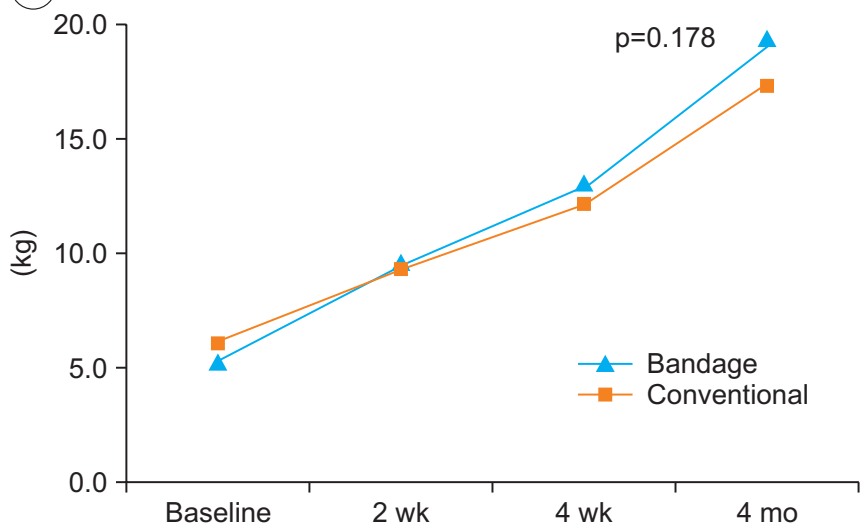

(D)

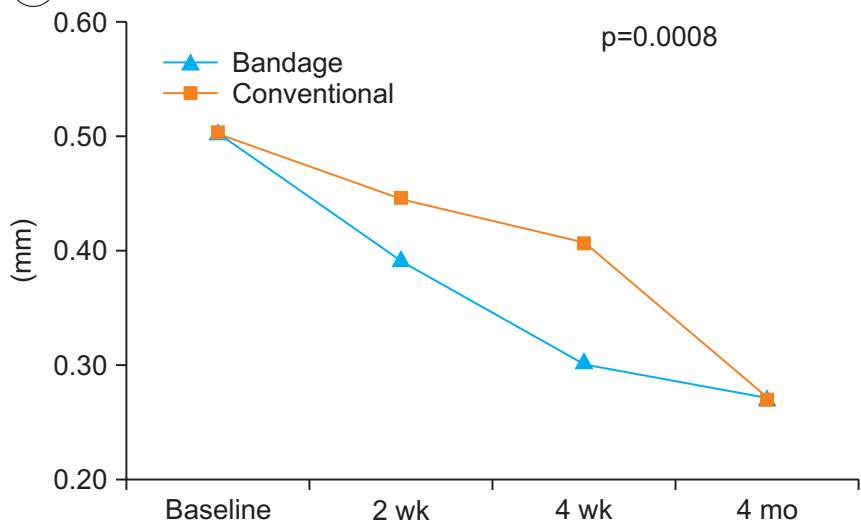

Fig. 4. On repeated-measures ANOVA, variables with significant time effect $(\mathrm{p}<0.05)$ and reciprocal effect $(\mathrm{p}<0.05)$ including figure-eight hand circumference $(A)$, Skin thickness (D), and visual analogue scale (VAS) (E). Moreover, variables with a significant time effect $(\mathrm{p}<0.05)$, but insignificant reciprocal effect $(p>0.05)$ include grasp power (B) and the Jebsen hand function test (C).

stantiate its use [14]. Salisbury et al. [15] and Ause-Ellias et al. [16] demonstrated a decrease in edema on burned hands using intermittent mechanical compression. Flowers [17] compared various wrapping techniques and massage for reducing hand edema secondary to a range of pathologies and found that edema was reduced in all cases, with the most effective method being a combination of compression wrap with continuous retrograde massage. In this study, we found early edema reduction 
in the modified hand compression bandage group and significant differences in time and reciprocal effects between the test and control groups.

A modified hand compression bandage was applied to the hand areas other than the finger joint. Thus, attempts were made not to restrict the finger joint ROM. Thus, we maximized the finger ROM exercise. In this study, a modified hand compression bandage showed a significant effect in improving the MCP joint ROM compared to the conventionally treated group. However, there was no difference in the PIP joint ROM.

Moreover, long-term outcomes showed a significant difference in MCP joint ROM. Post-burn hand edema induces the occurrence of fibrosis, thickening of the tissue, and gradually lowers tissue extendibility. This eventually leads to the decreased ROM $[18,19]$. Eventually, the decreased edema plays a key role in achieving a recovery of the ROM. Omar et al. [20] reported that electrical stimulation treatment was effective in improving edema, hand function, and ROM in patients with post-burn hand edema. Lowell et al. [19] reported that in patients with postburn both hand edema, following the treatment with Coban bandaging for the unilateral hand, showing that Coban bandaging was effective in improving the edema and finger joint ROM. The difference between the above studies and the current one is that previous studies have applied the total sum of MCP and PIP joint motion range in measuring the ROM. In this study, we measured each MCP and PIP joint and then analyzed them respectively. This study showed early reduction of edema resulting in MCP joint ROM improvement. However, the PIP joint was not effective for the reciprocal action effect.

This data suggests that a modified hand compression bandage treatment was more effective for the MCP joint than the PIP joint. The edema control had a negligible effect in improving the PIP joint ROM because a limited amount of fluid accumulated in the extracellular space owing to a smaller thickness of dermis and subcutaneous fat layer around PIP joint. In addition, the PIP joint is a difficult area to avoid bandaging, unlike the MCP joint area, because of variability in distance between the MCP joint and PIP joint among patients. These are considered to have effects in the improvement of ROM of the PIP joint.

Edema fluid in the extravascular space around the wound limits the flux of toxic metabolites and the exchange of vital nutrients, such as oxygen circulation around damaged tissues [21]. As described here, edema is associated with the healing process, inflammation and pain. Also in this study, there may be a relationship between the decreased edema and pain relief.

Burn scarring occurs in $70 \%$ of burn patients and pressure therapy has been widely used as the first line treatment for hypertrophic scars. A study conducted by Li-Tsang et al. [14] showed a significant difference in decreased skin thickness between treatment groups by examining pressure therapy in 26 burn patients. As with effects of pressure therapy, there was a decrease in cell density and myofibroblasts, leading to the impaired synthesis of collagen. Therefore, we infer that there is a decrease in the fluid influx to the interstitium. This leads to the speculation that there might have been effects on the edema, as well as, hypertrophic scaring. However, in our study, skin thickness assuming hypertrophic scar, showed no significant difference at the end of this study.

According to Covey et al. [22], grasp power in patients with post-hand burn, had a recovery of up to $60 \%$ and $42 \%$ compared with the non-affected side in the right and left hand, respectively. In this study, grasp power improved significantly with the time effect. However, there was no significant reciprocal action effect. Moreover, there was no significant time-dependent difference in the conventional treated group following the treatment.

Grasp power is associated with elbow, wrist, and hand functions. This leads us to speculate that the compression bandage did not completely reflect the functions of the hand in patients with post-burn edema [23]. The Jebsen hand function test also showed that there was a significant time effect. However, there was no significant reciprocal action effect. Furthermore, there was no significant time-dependent difference between the groups.

Schneider et al. [24] conducted a study showing that there were significant differences in the Jebsen hand function test between the post-burn rehabilitation group and the conventionally treated group. Likewise, in our study, there was a decrease in the Jebsen hand function test score following the initiation of rehabilitation therapy. However, there was no significant difference between the modified hand compression bandage group and the conventionally treated group.

Choi et al. [11] conducted a study where MCP Joint flexion orthoses were applied to patients' post-burn hands, reporting that there was an improvement in the ROM of 
the MCP Joint. However, there were no significant differences between grasp power and the Jebsen hand function test. In the MCP joint flexion orthoses group, there was significant improvement in the ROM of the MCP joint. However, there were no significant effects in improving the ROM of the PIP and distal interphalangeal (DIP) joint. Eventually, insignificant effects on the improvement of the ROM of the PIP and DIP joint were explained as a lack of significant differences in grasp power and the Jebsen hand function test.

King et al. [25] conducted a study to compare the effects between compression garments and compression bandaging in 21 patients with breast cancer-related lymphedema. They report the compression bandaging had increased effects on edema volume reduction. This leads to the speculation that the compression bandaging might be more effective for the treatment of lymphedema.

In patients with post-burn hand edema, there is a concurrent presence of lymphedema and hypertrophic scarring. Therefore, it would be difficult to draw a conclusion based on results obtained from patients with breast cancer-related lymphedema. According to this study, a modified hand compression bandage has positive effects to acute hand burns that are too sensitive for compression garments application. Further study on comparing the efficacy in the reduction of edema and improvement of hand function between compression garments and modified hand compression bandages to subacute hand burns is required to standardize the appropriate time and period to apply the modified hand compression bandage.

In conclusion, the modified hand compression bandage was effective to patients with post-burn hand edema, improving the ROM of MCP joint, hand circumference and skin thickness. The modified hand compression bandage will be clinically useful for the treatment of patients with post-hand burn edema.

\section{CONFLICT OF INTEREST}

No potential conflict of interest relevant to this article was reported.

\section{REFERENCES}

1. Smith MA, Munster AM, Spence RJ. Burns of the hand and upper limb: a review. Burns 1998;24:493-505.
2. Richard R, Staley M, Miller S, Warden G. To splint or not to splint: past philosophy and present practice. Part I. J Burn Care Rehabil 1996;17:444-53.

3. Frontera W, DeLisa JA. DeLisa's physical medicine and rehabilitation. 5th ed. Philadelphia: Saunders; 2010. p. 1125-50.

4. Moore ML, Dewey WS, Richard RL. Rehabilitation of the burned hand. Hand Clin 2009;25:529-41.

5. Atiyeh BS, El Khatib AM, Dibo SA. Pressure garment therapy (PGT) of burn scars: evidence-based efficacy. Ann Burns Fire Disasters 2013;26:205-12.

6. Dewey WS, Hedman TL, Chapman TT, Wolf SE, Holcomb JB. The reliability and concurrent validity of the figure-of-eight method of measuring hand edema in patients with burns. J Burn Care Res 2007;28:157-62.

7. Maihafer GC, Llewellyn MA, Pillar WJ Jr, Scott KL, Marino DM, Bond RM. A comparison of the figure-ofeight method and water volumetry in measurement of hand and wrist size. J Hand Ther 2003;16:305-10.

8. Leard JS, Breglio L, Fraga L, Ellrod N, Nadler L, Yasso $\mathrm{M}$, et al. Reliability and concurrent validity of the figure-of-eight method of measuring hand size in patients with hand pathology. J Orthop Sports Phys Ther 2004;34:335-40.

9. Kang TD, Jang KE, Park DS, Kim SB, Jung EH. Ultrasonographic assessment of nonsurgical treatment of postburn hypertrophic scar. J Korean Acad Rehabil Med 1999;23:397-404.

10. Jebsen RH, Taylor N, Trieschmann RB, Trotter MJ, Howard LA. An objective and standardized test of hand function. Arch Phys Med Rehabil 1969;50:311-9.

11. Choi JS, Mun JH, Lee JY, Jeon JH, Jung YJ, Seo CH, et al. Effects of modified dynamic metacarpophalangeal joint flexion orthoses after hand burn. Ann Rehabil Med 2011;35:880-6.

12. Vincent G, Radha H. burn rehabilitation. In: Braddom RL, editor. Physical medicine and rehabilitation, 4th ed. Philadelphia: Saunders; 2011. p. 1403-18.

13. Lund T, Onarheim H, Reed RK. Pathogenesis of edema formation in burn injuries. World J Surg 1992;16:29.

14. Li-Tsang CW, Feng B, Huang L, Liu X, Shu B, Chan YT, et al. A histological study on the effect of pressure therapy on the activities of myofibroblasts and keratinocytes in hypertrophic scar tissues after burn. Burns 2015;41:1008-16. 
15. Salisbury RE, Loveless S, Silverstein P, Wilmore DW, Moylan JA Jr, Pruitt BA Jr. Postburn edema of the upper extremity: evaluation of present treatment. J Trauma 1973;13:857-62.

16. Ause-Ellias KL, Richard R, Miller SF, Finley RK Jr. The effect of mechanical compression on chronic hand edema after burn injury: a preliminary report. J Burn Care Rehabil 1994;15:29-33.

17. Flowers KR. String wrapping versus massage for reducing digital volume. Phys Ther 1988;68:57-9.

18. Laseter GF. Management of the stiff hand: a practical approach. Orthop Clin North Am 1983;14:749-65.

19. Lowell M, Pirc P, Ward RS, Lundy C, Wilhelm DA, Reddy R, et al. Effect of 3M Coban Self-Adherent Wraps on edema and function of the burned hand: a case study. J Burn Care Rehabil 2003;24:253-8.

20. Omar MT, El-Badawy AM, Borhan WH, Nossier A. Improvement of edema and hand function in superficial second degree hand burn using electrical stimulation.
Egypt J Plast Reconstr Surg 2004;28:141-7.

21. Edgar DW, Fish JS, Gomez M, Wood FM. Local and systemic treatments for acute edema after burn injury: a systematic review of the literature. J Burn Care Res 2011;32:334-47.

22. Covey MH, Dutcher K, Heimbach DM, Marvin JA, Engrav LH, deLateur B. Return of hand function following major burns. J Burn Care Rehabil 1987;8:224-6.

23. Kong Y, Sohn S, Han J. A review study for grip strengths of hand. J Ergon Soc Korea 2010;29:715-25.

24. Schneider JC, Qu HD, Lowry J, Walker J, Vitale E, Zona M. Efficacy of inpatient burn rehabilitation: a prospective pilot study examining range of motion, hand function and balance. Burns 2012;38:164-71.

25. King M, Deveaux A, White H, Rayson D. Compression garments versus compression bandaging in decongestive lymphatic therapy for breast cancer-related lymphedema: a randomized controlled trial. Support Care Cancer 2012;20:1031-6. 